\title{
PROPOSIÇÃO DE ESTRUTURA FLEXÍVEL PARA MODELOS DE NEGÓCIO IOT
}

Tatiana D. Almeida (tatiana.almd@gmail.com) - Pós-Graduação em Engenharia de Produção, Universidade Federal de Santa Catarina - PPGEP-UFSC.

Jaime C. Pincheira (jcastill@uct.cl) - Ingeniería Industrial y de Sistemas / Universidad Catolica de Temuco - Chile.

Ângela M. F. Danilevicz (angeladanilevicz@gmail.com) - Universidade Federal do Rio Grande do Sul / Departamento de Engenharia de Produção

Diego Castro Fettermann (dcfettermann@gmail.com) - Pós-Graduação em Engenharia de Produção, Universidade Federal de Santa Catarina - PPGEP-UFSC.

\section{RESUMO}

O uso de tecnologias denominadas de Internet das Coisas (IoT) possibilita a integração e interação entre objetos por meio de sensores e conexões viabilizando a comunicação entre si e com o ambiente. Ao mesmo tempo que o IoT permite uma maior troca de dados, também torna mais complexa a construção dos modelos de negócio. Esta ampla rede de comunicação apresenta uma grande oportunidade para o desenvolvimento de negócios incluindo tecnologias IoT, ao mesmo tempo aumenta a complexidade para a construção dos modelos de negócio. Este estudo propõe uma estrutura flexível para modelos de negócio que utilizam tecnologias IoT. Foram propostas duas configurações customizáveis de modelos de negócio IoT, direcionadas a: manufatura e produtos/serviços. Como contribuições, este trabalho apresenta a identificação de padrões entre as proposições de modelos de negócio IoT, bem como a proposta customizável para estruturação de modelos para aplicação em negócios que utilizam as tecnologias IoT. Dessa forma, espera-se que gestores possam utilizar como ponto de partida as estruturas propostas para definir e/ou modificar seus modelos de negócio de acordo com o objetivo de seus negócios.

Palavras chave: Internet of Things; Modularidade; Análise de Cluster; 


\section{INTRODUÇÃ̃}

A Internet das Coisas (Internet of Things - IoT) promove a integração e interconexão entre uma grande variedade de objetos por meio de sensores e conexões à rede. Essa conexão tem o propósito de permitir que os objetos e rede interajam entre si e com o meio para que possam entender, monitorar e muitas vezes controlar o ambiente (NG; WAKENSHAW, 2017; FETTERMANN et al., 2018). São várias as tecnologias que envolvem as possibilidades de aplicação da IoT, tais como Radio Frequency Identification (ATZORI et al., 2010), sensores inteligentes (BORGIA, 2014), dispositivos móveis (PACHECO et al., 2016) e Wireless Sensor Networks (GUBBI et al., 2013). A incorporação destas tecnologias é crescente em diversos setores, bem como na agricultura (YU et al., 2010), em sistemas produto-serviço (ZANCUL et al., 2016), cidades inteligentes (HASHEM et al., 2016), manufatura (RYMASZEWSKA et al., 2017), saúde (FARAHANI et al., 2017), transporte (AFZAL et al., 2017), entre outros. É possível identificar que a quantidade de pesquisa que aborda o tema IoT é crescente e vem sendo um dos principais temas discutidos tanto nos setores empresariais quanto no ambiente acadêmico (JU et al., 2016).

Os modelos de negócio são apontados como um dos desafios mais significativos no que se refere à adoção das tecnologias IoT (MOHAMMADZADEH et al., 2018). Com o uso da IoT, verifica-se uma mudança voltada a uma perspectiva de ecossistema, tornando ineficiente a abordagem tradicional dos modelos de negócio, que apresentam uma perspectiva restrita ao nível de empresa (IKÄVALKO et al., 2018). Por apresentar uma infinidade de possibilidades de conexões, é preciso a adequação dos modelos de negócio para atender à complexidade trazida pela IoT (IKÄVALKO et al., 2018). A estrutura do ecossistema relacionado aos negócios não apresenta clareza devido à quantidade de soluções e os envolvidos não apresentam papéis definidos (WESTERLUND et al., 2014). A inclusão de soluções específicas de IoT geralmente atendem somente a algumas partes de problemas que requerem uma solução geral, o que gera uma fragmentação de problemas. Esse fator é uma das barreiras que justificam a estruturação de um modelo de negócio a partir da inclusão da IoT. Observa-se ainda que os gestores muitas vezes apresentam receio em realizar modificações em seus modelos de negócio (MARKENDAHL et al., 2017).

Devido a esta diversidade de possibilidades de aplicação das tecnologias IoT nos negócios (GHANBARI et al. 2017) e a possibilidade da aplicação do conceito de modularidade ao design organizacional (LANGLOIS, 2002), o objetivo deste estudo é propor uma forma de 
customizável de estruturação de modelos de negócio IoT. A proposta sugere uma estrutura mais flexível e adequada para a diversidade de aplicações que as tecnologias IoT disponibilizam para novos negócios. Com esta proposta, pretende-se que a estrutura possa ser utilizada como ponto de partida no desenvolvimento de novos modelos de negócio no contexto IoT e na modificação de modelos já existentes, de acordo com os objetivos dos negócios em questão.

\section{METODOLOGIA}

O método proposto para este trabalho é constituído de três etapas: (i) identificação das proposições de modelos de negócio IoT na literatura; (ii) levantamento das características das proposições de modelos de negócio IoT e identificação de padrões; e (iii) proposta customizável de estruturação para modelos de negócio no contexto IoT.

\subsection{Identificação das proposições de modelos de negócio IoT e identificação de padrões}

Para a primeira etapa, inicialmente foram definidos os eixos de pesquisa, sendo: internet das coisas (IoT) e demais definições, associadas ao tema de modelos de negócio. Foi realizada uma busca nas bases de dados acadêmicas Web of Science, Science Direct e Scopus restritos à títulos, palavras-chave e resumos. A busca foi realizada no período de 11 a 15 de junho de 2018, sem restrição temporal dos resultados nem quanto ao tipo de publicação.

No processo de filtragem as publicações foram analisadas quanto aos seguintes critérios: $(i)$ artigos duplicados; (ii) títulos alinhados ao tema de pesquisa e versão online disponível; (iii) resumos alinhados ao tema de pesquisa; (iv) apresentação de proposta ou descrição de modelo de negócio para o contexto IoT; e $(v)$ propostas de modelos de negócio IoT estruturadas por blocos de construção. O objetivo dos critérios de seleção foi identificar os estudos primários relevantes que de fato apresentam propostas ou descrições de modelos de negócio IoT estruturados de forma semelhante, para o atendimento ao objetivo da pesquisa. Primeiramente, foram analisadas as estruturas das proposições dos modelos de negócio IoT levantadas. Observou-se que a maior parte das proposições $(87,5 \%)$ identificadas na literatura são estruturadas por blocos de construção e, por este motivo, estes foram os casos considerados neste trabalho.

2.2 Levantamento das características das proposições de modelos de negócio IoT e identificação de padrões 
No levantamento, foi realizada uma análise de conteúdo no sentido de agrupar os nomes de blocos semelhantes que apresentam o mesmo direcionamento no modelo de negócio IoT. Foram identificados 84 blocos de construção e após esta análise de conteúdo foi possível agrupar por similaridade diversos blocos de construção, resultando em um total de 41 diferentes blocos. Os dados foram organizados na forma de uma matriz associando $i$ proposições de modelos por blocos $I_{28}^{1}$ e $j$ blocos de construção $J_{41}^{1}$ (Figura 1).

Figura 1 - Estrutura de banco de dados coletados em propostas de modelos de negócio IoT

\begin{tabular}{|c|c|c|c|c|c|c|c|c|c|c|c|c|c|c|c|c|}
\hline $\begin{array}{ll}\text { Modelos } & \text { Blocos } \\
\end{array}$ & $j_{1}$ & $j_{2}$ & $j_{3}$ & $j_{4}$ & $j_{5}$ & $j_{6}$ & $j_{7}$ & $j_{8}$ & $j_{9}$ & $\boldsymbol{j}_{10}$ & $j_{11}$ & $j_{12}$ & $j_{13}$ & $j_{14}$ & $\cdots$ & $j_{41}$ \\
\hline$i_{1}$ & 0 & 0 & 1 & 0 & 0 & 0 & 0 & 0 & 0 & 0 & 0 & 1 & 0 & 1 & $\ldots$ & 0 \\
\hline$i_{2}$ & 0 & 0 & 0 & 0 & 0 & 0 & 0 & 0 & 0 & 0 & 0 & 1 & 0 & 1 & $\ldots$ & 0 \\
\hline$i_{3}$ & 0 & 0 & 1 & 0 & 0 & 0 & 0 & 0 & 0 & 0 & 0 & 1 & 0 & 1 & $\ldots$ & 0 \\
\hline$i_{4}$ & 0 & 0 & 1 & 0 & 0 & 0 & 1 & 0 & 0 & 0 & 0 & 1 & 0 & 1 & $\ldots$ & 0 \\
\hline$i_{5}$ & 0 & 0 & 1 & 0 & 0 & 0 & 0 & 0 & 0 & 0 & 0 & 1 & $\underline{0}$ & 1 & 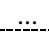 & 0 \\
\hline$i_{6}$ & 0 & 0 & 1 & 0 & 0 & 0 & 1 & 0 & 0 & 0 & 0 & 1 & 0 & 1 & $\ldots$ & 0 \\
\hline$i_{7}$ & 0 & 0 & 1 & 0 & 0 & 0 & 0 & 0 & 0 & 0 & 0 & 1 & 0 & 1 & $\ldots$ & 0 \\
\hline$i_{8}$ & 0 & 0 & 0 & 0 & 0 & 0 & 0 & 0 & 0 & 0 & 0 & 0 & 0 & 0 & $\ldots$ & 0 \\
\hline$i_{9}$ & 0 & 0 & 1 & 0 & 0 & 0 & 0 & 0 & 0 & 0 & 0 & 1 & 0 & 1 & $\ldots$ & 0 \\
\hline$i_{10}$ & 0 & 0 & 1 & 0 & 0 & 0 & 0 & 0 & 0 & 0 & 0 & 1 & 0 & 1 & $\ldots$ & 0 \\
\hline$\ldots$ & & & & & $\ldots$ & . & & . & 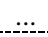 & $\ldots$ & 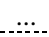 & .:- & $\ldots$ & $\ldots$ & $\ldots$ & \\
\hline$i_{28}$ & 0 & 0 & 1 & 0 & 0 & 0 & 0 & 0 & 0 & 0 & 0 & 1 & 0 & 1 & $\ldots$ & 0 \\
\hline
\end{tabular}

Neste trabalho, a Análise Multivariada de Agrupamentos (Cluster) foi realizada de forma a identificar agrupamentos de $i$ proposições de modelos IoT que apresentem uma estrutura de $j$ blocos de construção semelhantes entre si. Além disso, foram identificados os padrões de aplicação das proposições. O método de análise de agrupamento utilizado neste trabalho foi o método hierárquico com a medida de similaridade de Jaccard (MILLIGAN; COOPER, 1986), em razão da característica binária dos dados e o interesse exclusivo em ocorrências em que a proposição $i$ está associada aos blocos de construção $j$. A técnica de agrupamento hierárquico faz uma ligação das amostras por suas associações, gerando um dendograma em que as amostras que se assemelham são agrupadas entre si. Foi realizada a análise de agrupamento hierárquica dos dados utilizando o método de armazenamento em cluster centroide. Este método refere-se ao algoritmo no qual a similaridade entre agrupamentos é medida como a distância entre os centroides de agrupamentos (RENCHER, 2003). A utilização deste método foi selecionada em razão de ser menos afetada por condições de outliers em comparação a outros métodos hierárquicos (RENCHER, 2003), sendo que na amostra existem casos muito específicos. A análise dos dados foi realizada com a utilização dos softwares Microsoft Excel $2013^{\circledR}$, para a organização dos dados, e o IBM SPSS Statistics $23^{\circledR}$ para a análise de agrupamento. A definição da quantidade de agrupamentos é realizada por meio de análise do 
dendograma e da coesão entre as ocorrências e separação entre os agrupamentos, definidas pelo índice de Silhouette (ROUSSEUW, 1987). Após a identitificação da quantidade de agrupamentos que expressam uma maior homogeneidade interna e heterogeneidade entre os mesmos foi realizada a análise de agrupamentos $k$-means para o agrupamento das proposições de modelos de negócio IoT. O método de agrupamento k-means é um método usado para particionar automaticamente um conjunto de dados em $k$ grupos (WAGSTAFF et al., 2001). Por meio desta análise, observa-se se os objetos dentro de um agrupamento são de fato semelhantes aos outros inseridos no mesmo agrupamento (homogeneidade), e diferentes de outros objetos identificados em outros agrupamentos (denotando heterogeneidade) (RENCHER, 2003). Neste trabalho, o resultado desta análise busca identificar as proposições semelhantes entre si de acordo com a suas estruturas de blocos propostas. Este resultado permite identificar a possibilidade de padrões genéricos de modelos de negócio IoT de acordo com sua área de aplicação. A partir da análise do dendograma (Figura 2) e o valor do coeficiente de Silhouette foi identificado que a identificação de dois grupamentos a mais adequada para os dados analisados.

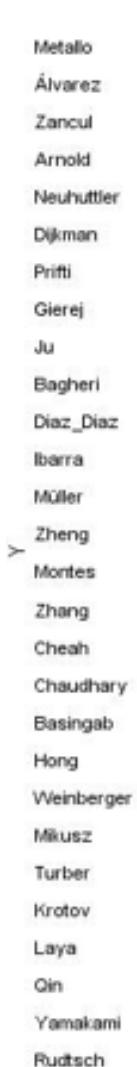

Figura 2 - Dendograma resultante da análise de agrupamento

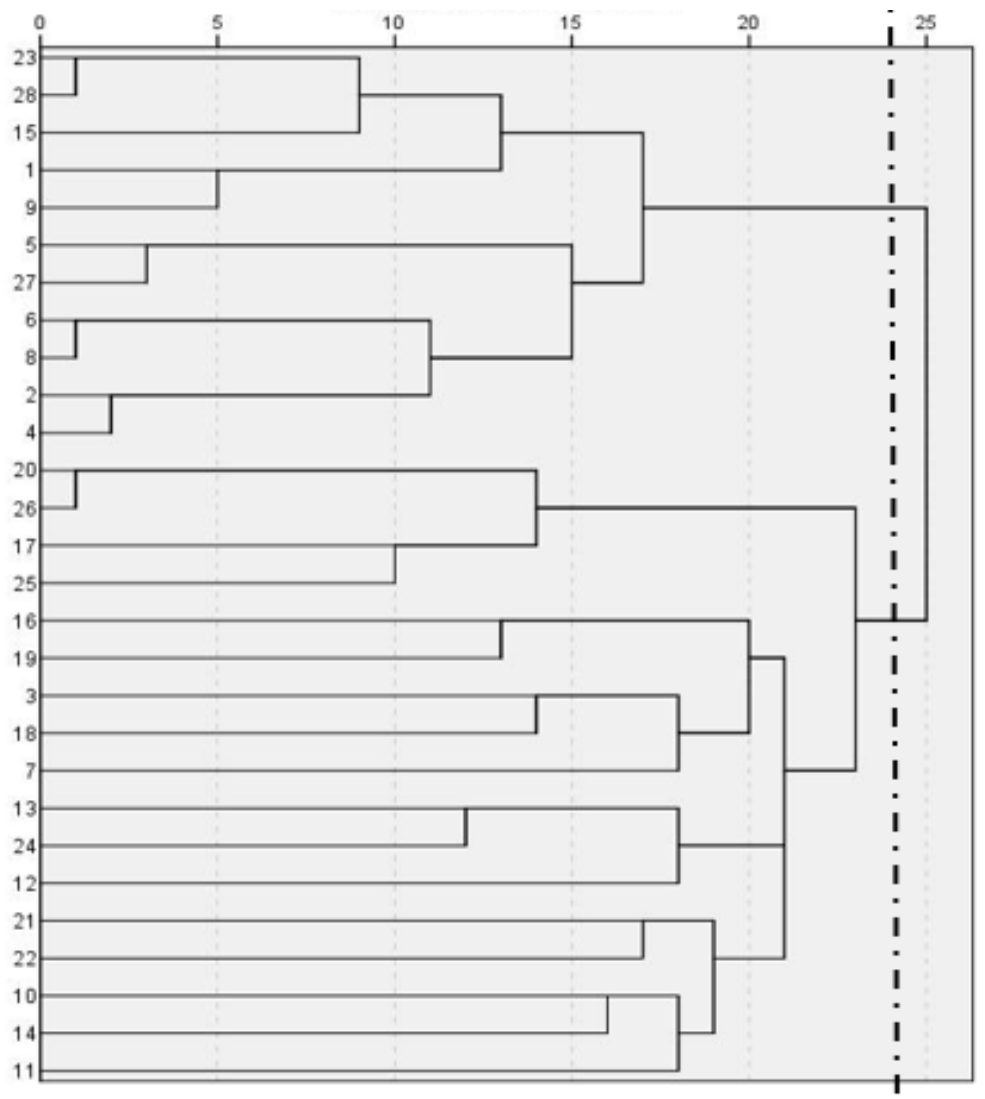


Fonte: $\operatorname{SPSS}^{\circledR}$ v.23

\subsection{Proposta customizável de estruturação para modelos de negócio no contexto IoT}

A partir da definição dos padrões de aplicação na análise das proposições de modelos de negócio IoT, cada agrupamento é analisado separadamente com o intuito de identificar em cada um deles uma plataforma de blocos de construção. Para tanto, é utilizada uma análise da frequência dos blocos de construção em cada um dos agrupamentos identificados. Propõe-se calcular a média considerando as frequências totais de cada bloco, sendo os blocos com frequências acima da média total são considerados integrantes da plataforma. Os demais blocos são agrupados para sua utilização a partir de especificidades e necessidades de cada negócio.

\section{PROPOSTA CUSTOMIZÁvel DE ESTRUTURAÇÃo PARA MODELOS DE NEGÓCIO}

A partir dos resultados e após análise dos agrupamentos identificados e as características dos modelos de negócios IoT agrupados, estes padrões foram denominados de acordo com o foco a que se referem seus modelos de negócio (Quadro 1). O primeiro padrão identificado concentra em sua maioria proposições de modelos de negócio com direcionamento à manufatura, enquanto o segundo à produtos e/ou serviços. É válido ressaltar que três proposições não apresentaram direcionamento, mas sim abordagens genéricas de aplicação de modelos de negócio IoT (HONG, 2016; BASINGAB et al., 2017; KROTOV, 2017). Todavia, na análise de agrupamentos realizada, estes casos foram agrupados no padrão Manufatura.

Quadro 1 - Direcionamento das propostas de modelos de negócio IoT

\begin{tabular}{ll}
\hline Direcionamento & \multicolumn{1}{c}{ Referências } \\
\hline \multirow{3}{*}{ Manufatura } & Qin e Yu (2015); Rudtsch et al. (2014); Turber e Smiela (2014); Chaudhary et al. (2015); Arnold \\
& et al. (2016); Weinberger et al. (2016); Cheah e Wang (2017); Gierej (2017); Mikusz et al. \\
& (2017); Montes (2017); Yamakami (2017); Zhang e Wen (2017); Ibarra et al. (2018); Müller et \\
& al. (2018). \\
& Álvarez et al. (2015); Dijkman et al. (2015); Ju et al. (2016); Zancul et al. (2016); Bagheri e \\
Produto/Serviço & $\begin{array}{l}\text { Movahed (2017); Diaz-Diaz et al. (2017); Neuhüttler et al. (2017); Prifti et al. (2017); Zheng et } \\
\text { al. (2017); Laya et al. (2018); Metallo et al. (2018). }\end{array}$ \\
\hline
\end{tabular}

Posteriormente, os padrões passaram por análises independentes para proposta customizável de estruturação para modelos de negócio IoT. Com base na análise da similaridade entre as 28 proposições identificadas na literatura foram propostas duas estruturas para modelos de negócio IoT direcionados à dois tipos de aplicação: em manufatura e em produtos/serviços. Ambas propostas possuem a característica de serem customizáveis. Cada negócio possui suas 
determinadas especificidades independentemente do setor ao qual está inserido. Por este motivo, as estruturas propostas neste trabalho podem ser customizadas de acordo com as necessidades e objetivos específicos do negócio.

As propostas apresentadas neste trabalho apresentam a seguinte estrutura: plataforma e categorias de blocos de construção. A plataforma sugere uma estrutura base para desenvolvimento do modelo de negócio IoT, ou seja, a estrutura inicial genérica para os modelos de negócio para cada um dos direcionamentos ao qual o negócio se insere (manufatura ou produtos/serviços). Posteriormente, podem ser acoplados nesta plataforma quaisquer outros blocos de construção remanescentes de acordo com a necessidade específica do caso. Neste estudo, estes blocos estão organizados de acordo com seu direcionamento principal a fim de facilitar a decisão de gestores conforme os objetivos de seus negócios. Dessa forma, o modo de estruturação do modelo de negócio IoT final será o resultado de uma estrutura de plataforma + blocos de construção determinados de acordo com especificidades e/ou objetivos do negócio.

\subsection{Proposta customizável direcionada à Manufatura}

No padrão de modelos de negócio referente à aplicação voltada à Manufatura, foram identificados nove blocos com frequência acima da média, sendo estes: fluxo de receita, proposição de valor, clientes, atividades e processos chave, dados, arquitetura digital, stakeholders, objetos inteligentes e recursos chave.

Posteriormente, foram analisados os blocos de construção restantes, identificando similaridades entre suas aplicações com o objetivo de se identificar categorias de blocos associados. O objetivo da proposta das categorias de blocos de construção é apresentar conjuntos que possam ser customizados de acordo com as especificidades e necessidades de cada negócio. Como estes blocos apresentaram uma frequência reduzida em relação aos blocos considerados na plataforma, entende-se que estes blocos podem ser necessários para características específicas de negócio. Sendo assim, ao estruturar seu modelo de negócio, a empresa pode adaptar as categorias de blocos à plataforma a partir de seus objetivos e visão de negócio. A seguir a estrutura proposta é apresentada (Figura 3). 
Figura 3 - Proposta customizável de estruturação para modelo de negócio IoT direcionado à Manufatura
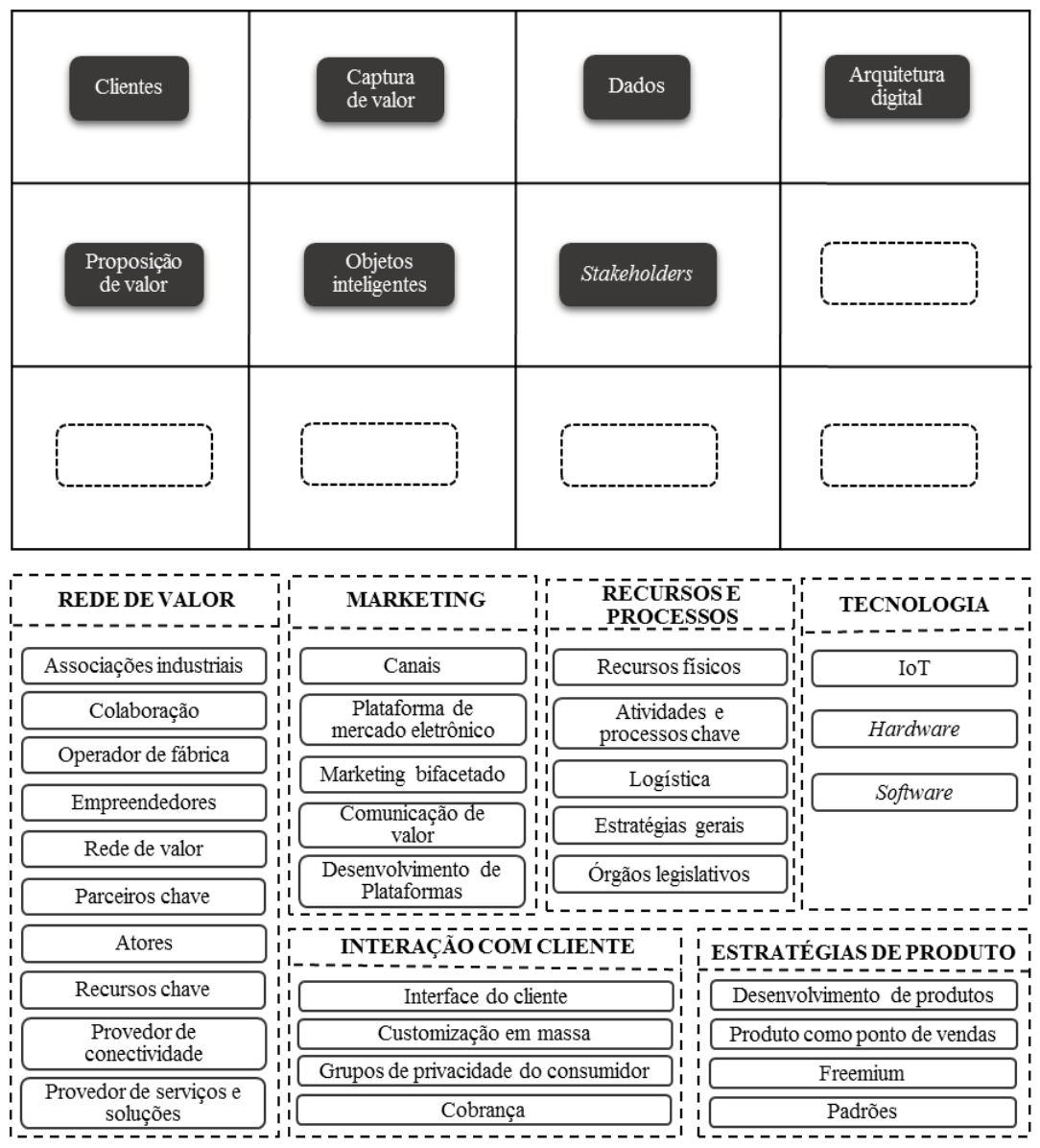

\subsection{Proposta customizável direcionada a Produtos/Serviços}

Assim como no primeiro padrão de modelos de negócio IoT, houve o intuito de definir uma plataforma de blocos de construção para o segundo padrão, para modelos de negócio IoT direcionados à área de produtos e/ou serviços. Diferente do primeiro padrão, em que foi observado um número superior de blocos remanescentes, no segundo padrão apenas três blocos foram identificados. Isso significa que o grupo de proposições de modelos direcionados à produtos/serviços é muito mais homogêneo entre as proposições analisadas. Sendo assim, restaram apenas três opções para adição à plataforma base. Estes blocos apresentam direcionados ao desenvolvimento de produtos especificamente, cadeia de valor e ações voltadas à responsabilidade social (Figura 4). 
Figura 4 - Proposta customizável de estruturação para modelo de negócio IoT direcionado à Produtos/Serviços

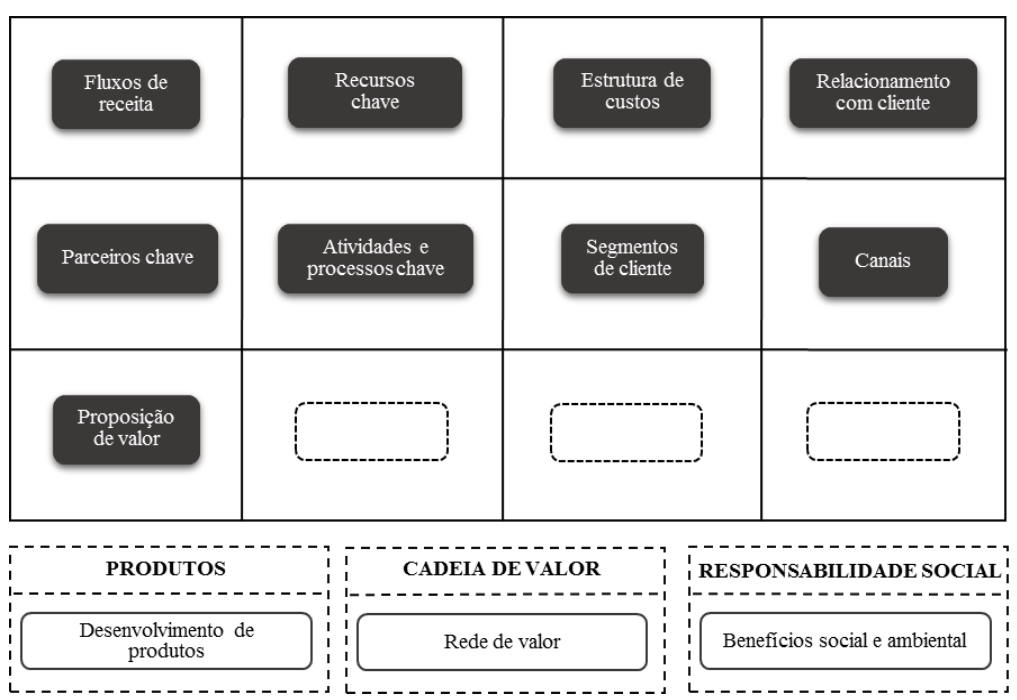

\section{CONCLUSÃO}

Por meio da análise de proposições de modelos de negócio IoT identificadas na literatura pôdese observar que existe uma grande variedade de aplicações destas proposições. Verificou-se uma limitação da aplicação prática genérica destas proposições, em razão de que muitas referem-se a casos de aplicação muito específicos, tais como manutenção preditiva no âmbito industrial (BASINGAB et al., 2017), uso de plataforma inteligente voltado ao social care (LAYA et al., 2018), oferta de serviços no setor de educação por meio de plataforma Educação como Serviço (EAAS) (PRIFTI et al., 2017). Essas especificidades dificultam a utilização destas proposições de modelos de negócio IoT em casos mais genéricos de negócio. Por outro lado, percebe-se ainda que existem propostas mais genéricas na literatura, porém seu uso fica insuficiente quando consideradas as particularidades de negócios específicos. Dessa forma, como contribuição teórica, este trabalho apresenta uma análise destes casos de forma a propor uma forma de estruturação customizável, utilizando o conceito de configuração modular aplicada no âmbito organizacional. Essa proposta pode ser utilizada como ponto de partida para a estruturação de um modelo de negócio IoT direcionado à manufatura ou produtos/serviços, considerando uma plataforma que apresenta blocos de construção considerados genéricos para a área, mais agrupamentos de blocos que podem ser inseridos de acordo com as especificidades no negócio em questão. Como contribuição prática este trabalho apresenta duas propostas customizáveis de estruturação para modelos de negócio IoT. Estas propostas podem ser utilizadas pelos gestores conforme o direcionamento de seu negócio. A estrutura segue a 
configuração de plataforma + categorias de blocos de construção, em que a plataforma consiste no ponto de partida em uma das etapas de modificações ou estruturações em seus modelos de negócio. Posteriormente, pode-se acoplar conforme necessidade e objetivo quaisquer dos blocos agrupados nas categorias de blocos de construção propostas. Recomenda-se para futuras pesquisas em relação ao tema, a aplicação da proposta a fim de testar a real eficiência deste tipo de modelo de negócio em empresas que incorporam tecnologias IoT.

\section{AGRADECIMENTO}

"O presente trabalho foi realizado com apoio da Coordenação de Aperfeiçoamento de Pessoal de Nível Superior -Brasil (CAPES) - Código de Financiamento 001

\section{REFERÊNCIAS}

AFZAL, B.; ALVI, S. A.; SHAH, G. A.; MAHMOOD, W. Energy Efficient Context Aware Traffic Scheduling for IoT Applications. Ad Hoc Networks, v. 62, p. 101-115, 2017.

ALVAREZ, O.; GHANBARI, A.; MARKENDAHL, J. Smart Energy: Competitive Landscape and Collaborative Business Models. Proceedings of the 18th International Conference on Intelligence in Next Generation Networks (ICIN), p. 114-120, 2015.

ARNOLD, C.; KIEL, D.; VOIGT, K. How the Industrial Internet of Things Changes Business Models in Different Manufacturing Industries. International Journal of Innovation Management, v. 20, n. 08, p. 1640015, 2016.

ATZORI, L.; IERA, A.; MORABITO, G. The Internet of Things: A Survey. Computer Networks, v. 54, n. 15, p. $2787-2805,2010$.

BAGHERI, M.; MOVAHED, S. H. The Effect of the Internet of Things (IoT) on Education Business Model. Proceedings of the 12th International Conference on Signal-Image Technology \& Internet-Based Systems (SITIS), p. 435-441, 2016.

BASINGAB, M.; RABELO, L.; NAGADI, K.; ROSE, C.; GUTIÉRREZ, E.; JUNG, W. I. Business Modeling Based on Internet of Things: A Case Study of Predictive Maintenance Software Using ABS Model. Proceedings of the Second International Conference on Internet of things and Cloud Computing, p. 11. ACM, 2017.

BORGIA, E. The Internet of Things Vision: Key Features, Applications and Open Issues. Computer Communications, v. 54, p. 1-31, 2014.

CHAUDHARY, R.; PANDEY, J.; PANDEY, P. Business Model Innovation through Big Data. Proceedings of the International Conference on Green Computing and Internet of Things (ICGCIoT), p. 259-263, 2015.

CHEAH, S.; WANG, S. Big Data-Driven Business Model Innovation by Traditional Industries in the Chinese Economy.Journal of Chinese Economic and Foreign Trade Studies, v. 10, n. 3, p. 229-251, 2017. 
DÍAZ-DÍAZ, R.; MUÑOZ, L.; PÉREZ-GONZÁLEZ, D. Business Model Analysis of Public Services Operating in the Smart City Ecosystem: The Case of SmartSantander. Future Generation Computer Systems, 2017.

DIJKMAN, R.; SPRENKELS, B.; PEETERS, T.; JANSSEN, A. Business Models for the Internet of Things. International Journal of Information Management, v. 35, n. 6, p. 672-678, 2015.

FARAHANI, B.; FIROUZI, F.; CHANG, V.; BADAROGLU, M.; CONSTANT, N.; MANKODIYA, K. Towards Fog-Driven IoT e-Health: Promises and Challenges of IoT on Medicine and Healthcare. Future Generation Computer Systems, 2017.

FETTERMANN, D. C.; CAVAlCANTE, C. G. S.; AlMEIDA, T. D. D.; TORTORELlA, G. L. (2018). How does Industry 4.0 contribute to operations management? Journal of Industrial and Production Engineering, v.35, n.4, 255-268, 2018 .

GHANBARI, A.; LAYA, A.; ALONSO-ZARATE, J.; MARKENDAHL, J. Business Development in the Internet of Things: A Matter of Vertical Cooperation. IEEE Communications Magazine, v. 55, n. 2, p. 135-141, 2017.

GIEREJ, S. The Framework of Business Model in the Context of Industrial Internet of Things. Procedia Engineering, v. 182, p. 206-212, 2017.

GUBBI, J.; BUYYA, R.; MARUSIC, S.; PALANISWAMI, M. Internet of Things (IoT): A Vision, Architectural Elements, and Future Directions. Future Generation Computer Systems, v. 29, n. 7, p. 1645-1660, 2013.

HASHEM, I.; CHANG, V.; ANUAR, N.; ADEWOLE, K.; YAQOOB, I.; GANI, A.; ...; CHIROMA, H.. The Role of Big Data in Smart City. International Journal of Information Management, v. 36, n. 5, p. 748-758, 2016.

HONG, H. Analysis of Business Framework for Internet of Things. Indian Journal of Science and Technology, v. 9 , n. 37, 2016.

IBARRA, D.; GANZARAIN, J.; IGARTUA, JI. Business Model Innovation through Industry 4.0: A Review. Procedia Manufacturing, v. 22, p. 4-10, 2018.

IKÄVALKO, H.; TURKAMA, P.; SMEDLUND, A. Value Creation in the Internet of Things: Mapping Business Models and Ecosystem Roles. Technology Innovation Management Review, v. 8, n. 3, 2018.

JU, J.; KIM, M.-S.; AHN, J.-H. Prototyping Business Models for IoT Service. Procedia Computer Science, v. 91, p. 882-890, 2016.

KROTOV, V. The Internet of Things and New Business Opportunities. Business Horizons, v. 60, n. 6, p. 831$841,2017$.

LANGLOIS, R. N. Modularity in Technology and Organization. Journal of Economic Behavior \& Organization, v. 49, n. 1, p. 19-37, 2002.

LAYA, A.; MARKENDAHL, J.; LUNDBERG, S. Network-Centric Business Models for Health, Social Care and Wellbeing Solutions in the Internet of Things. Scandinavian Journal of Management, v. 34, n. 2, p 103-116, 2018. 
MARKENDAHL, J.; LUNDBERG, S.; KORDAS, O.; MOVIN, S. On the Role and Potential of IoT in Different Industries: Analysis of Actor Cooperation and Challenges for Introduction of New Technology. Internet of Things Business Models, Users, and Networks, p. 1-8. IEEE, 2017.

METALlO, C.; AGRIFOGLIO, R.; SCHIAVONE, F.; MUELLER, J. Understanding Business Model in the Internet of Things Industry. Technological Forecasting and Social Change, 2018.

MIKUSZ, M.; SCHÄFER, T.; TARABA, T.; JUD, C. Transforming the Connected Car into a Business Model Innovation. Proceedings of the 19th IEEEConference on Business Informatics (CBI), p. 247-256, 2017.

MILLIGAN, G. W.; COOPER, M. C. A Study of the Comparability of External Criteria for Hierarchical Cluster Analysis. Multivariate Behavioral Research, v. 21, n. 4, p. 441-458, 1986.

MOHAMMADZADEH, A.; GHAFOORI, S.; MOHAMMADIAN, A.; MOHAMMADKAZEMI, R.; MAHBANOOEI, B.; GHASEMI, R. A Fuzzy Analytic Network Process (FANP) Approach for Prioritizing Internet of Things Challenges in Iran. Technology in Society, 2018.

MONTES, J.O. Impacts of 3D Printing on the Development of New Business Models. Proceedings of the IEEE European Technology and Engineering Management Summit (E-TEMS), 2016.

MÜLLER, J. M.; BULIGA, O.; VOIGT, K. Fortune Favors the Prepared: How SMES Approach Business Model Innovations in Industry 4.0. Technological Forecasting and Social Change, v. 132, p. 2-17, 2018.

NEUHÜTTLER, J.; WOYKE, I. C.; GANZ, W. Applying Value Proposition Design for Developing Smart Service Business Models in Manufacturing Firms. Proceedings of the International Conference on Applied Human Factors and Ergonomics, p. 103-114, 2017.

NG, I. C. L.; WAKENSHAW, S. Y. L. The Internet-of-Things: Review and Research Directions. International Journal of Research in Marketing, v. 34, n. 1, p. 3-21, 2017.

PACHECO, F.; KLEIN, A.; RIGHI, R. Modelos de Negócio para Produtos e Serviços Baseados em Internet das Coisas: Uma Revisão da Literatura e Oportunidades de Pesquisas Futuras. REGE - Revista de Gestão, v. 23, n. 1, p. 41-51, 2016.

PRIFTI, L.; KNIGGE, M.; LÖFFLER, A.; HECHT, S.; KRCMAR, H. Emerging Business Models in Education Provisioning: A Case Study on Providing Learning Support as Education-as-a-Service. International Journal of Engineering Pedagogy (iJEP), v. 7, n. 3, p. 92-108, 2017.

QIN, Q.; YU, H. Research on the Internet of Things Business Model of Telecom Operators Based on the Value Net. Management \& Engineering, n. 21, p. 8, 2015.

RENCHER, A. C. Methods of Multivariate Analysis. John Wiley \& Sons, 2003.

ROUSSEEUW, P. J. Silhouettes: A Graphical Aid to the Interpretation and Validation of Cluster Analysis. Journal of Computational and Applied Mathematics, v. 20, p. 53-65, 1987. 
RUDTSCH, V.; GAUSEMEIER, J.; GESING, J.; MITTAG, T.; PETER, S. Pattern-based Business Model Development for Cyber-Physical Production Systems. Procedia CIRP, v. 25, p. 313-319, 2014.

RYMASZEWSKA, A.; HELO, P.; GUNASEKARAN, A. IoT powered servitization of manufacturing-an exploratory case study. International Journal of Production Economics, 2017.

TURBER, S.; SMIELA, C. A Business Model Type for the Internet of Things. Proceedings of the 22nd European Conference on Information Systems, 2014.

WAGSTAFF, K.; CARDIE, C.; ROGERS, S.; SCHRÖDL, S. Constrained k-means Clustering with Background Knowledge. ICML, v.1, p.577-584, 2001.

WEINBERGER, M.; BILGERI, D.; FLEISCH, E. IoT Business Models in an Industrial Context. Automatisierungstechnik, v. 64, n. 9, p. 699-706, 2016.

WESTERLUND, M.; LEMINEN, S.; RAJAHONKA, M. Designing Business Models for the Internet of Things. Technology Innovation Management Review, v. 4, n. 7, 2014.

YAMAKAMI, T. A 3-Dimensional View Model of IoT-Empowered Business Model Engineering Towards Borderless Business Reengineering. Proceedings of the 19th International Conference on Advanced Communication Technology (ICACT), p. 690-694, 2017.

YU, L.; XUEMEI, L.; JIAN, Z.; YUNING, X. Research on the Innovation of Strategic Business Model in Green Agricultural Products Based on Internet of Things (IoT). Proceedings of the 2nd International Conference on E-business and Information System Security, 2010.

ZANCUL, E.; TAKEY, S.; BARQUET, A.; KUWABARA, L.; CAUCHICK MIGUEL, P.; ROZENFELD, H. Business Process Support for IoT Based Product-Service Systems (PSS). Business Process Management Journal, v. 22, n. 2, p. 305-323, 2016.

ZHANG, Y.; WEN, J. The IoT Electric Business Model: Using Blockchain Technology for the Internet of Things. Peer-to-Peer Networking and Applications, v. 10, n. 4, p. 983-994, 2017.

ZHENG, M.; MING, X.; WANG, L.; YIN, D.; ZHANG, X. Status Review and Future Perspectives on the Framework of Smart Product Service Ecosystem. Procedia CIRP, v. 64, n. 1, p. 181-186, 2017. 